
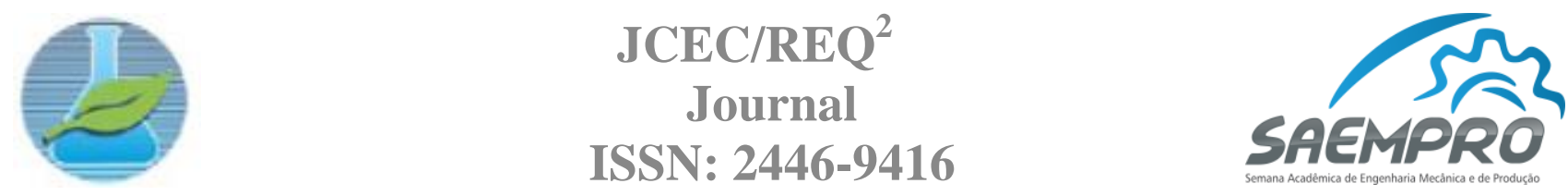

\author{
"EU, A INDÚSTRIA E O MUNDO" \\ 08 a 11 de novembro de 2016 no campus Viçosa da UFV \\ Departamento de Engenharia de Produção e Mecânica - DEP \\ Universidade Federal de Viçosa - UFV
}

\title{
INFLUENCIA DA VELOCIDADE DE CORTE DO PROCESSO DE TORNEAMENTO NOS RESULTADOS DE RUGOSIDADE DO AÇO ABNT 1020
}

\author{
Thiago Rosado de Paula, Lucas Benini \\ Universidade Federal de Viçosa, Departamento de Engenharia de Produção e Mecânica \\ $\mathrm{Ph}$. Rolfs s/n - 36570-000 - Viçosa - MG \\ thiago.rosado@ufv.br, lucas.benini@ufv.br
}

\section{INTRODUÇÃO}

De acordo com Amorim (2002) em quase todos os produtos é necessário o uso de procedimentos de usinagem em algum momento da sua fabricação, sendo difícil citar algum produto que não demande um processo de usinagem de forma direta ou indireta. $\mathrm{O}$ alto grau de precisão das dimensões, o bom acabamento superficial e a grande variedade de geometrias possíveis de serem usinadas justificam a grande utilização dos processos de usinagem. Essas características fazem com que os demais processos de fabricação dificilmente consigam substituir a usinagem.

Além disto, este processo de fabricação é muitas vezes empregado com a intuito de promover uma melhora do acabamento superficial ou tolerância dimensional do produto manufaturado por outros processos de fabricação. Este trabalho tem o objetivo de verificar a influência dos parâmetros de processo nos resultados de trabalho de torneamento do aço ABNT 1020.

\section{METODOLOGIA}

O procedimento foi realizado no Laboratório de Usinagem do Departamento de Engenharia Mecânica da UFV. Depois preparação do corpo de prova, este foi fixado na máquina-ferramenta e definidas as condições de corte (Tab. 1).

Após o término do percurso de avanço, mensurou-se o diâmetro da peça e os resultados dos valores de rugosidade de $\mathrm{Ra}$ e $\mathrm{Rz}$ da superfície da peça. Esses dois parâmetros de rugosidade foram mensurados em cinco pontos distintos da respectiva superfície usinada e realizada uma média destes resultados para caracterizar a rugosidade da amostra. A velocidade de corte foi variada durante os ensaios, assim como o avanço e a profundidade de corte. Isso foi realizado buscando-se avaliar a influência destes parâmetros na usinagem do aço ABNT 1020.

\section{RESULTADOS}

Considerando a velocidade de corte abaixo da velocidade máxima na qual a ferramenta suporta, $330 \mathrm{~m} / \mathrm{min}$, a profundidade de corte selecionada foi de $2,0 \mathrm{~mm}$ (considerada otimizada pelo fabricante) e o avanço selecionado foi maior que $0,23 \mathrm{~mm} / \mathrm{ver}$ (recomendado pelo fabricante), conforme apresenta a Tab. 1.

Neste ensaio observou-se que os parâmetros de rugosidade média ultrapassaram os valores considerados aceitáveis para o aço ABNT $1020(\mathrm{Ra}=12,5 \mu \mathrm{m})$, conforme observado na Tab. 1. Em um novo ensaio utilizou-se a velocidade de corte $\mathrm{Vc}=179,79 \mathrm{~m} / \mathrm{min}$, a qual está abaixo da velocidade de corte máxima suportada pela ferramenta segundo recomendações do fabricante. 
Tabela 2: Avaliação da velocidade de corte.

\begin{tabular}{|c|c|c|c|c|c|}
\hline Passe & $\mathbf{V c}(\mathbf{m} / \mathbf{m i n})$ & $\mathbf{a p}(\mathbf{m m})$ & $\mathbf{f}(\mathbf{m m} / \mathbf{r e v})$ & $\mathbf{R a}(\boldsymbol{\mu m})$ & $\mathbf{R z}(\boldsymbol{\mu m})$ \\
\hline 1 & 185,04 & 2 & 0,348 & 23,87 & 100,83 \\
\hline 2 & 182,07 & 2 & 0,348 & 23,49 & 100,31 \\
\hline
\end{tabular}

Foram adotados os valores de profundidade de corte considerada ótima pelo fabricante (ap = $2,0 \mathrm{~mm}$ ) e o avanço próximos do considerado ótimo pelo fabricante ( $\mathrm{f}=0,18 \mathrm{~mm} / \mathrm{ver}$ ) respectivamente. Neste ensaio, conseguiu-se realizar 16 passes de usinagem para que o valor de $\mathrm{Ra}$ ultrapassasse o valor máximo admitido. A Tab. 2 apresenta os valores dos parâmetros de rugosidade para as respectivas condições de ensaios.

Tabela 2: Avaliação da velocidade de corte.

\begin{tabular}{|c|c|c|c|c|c|}
\hline Passe & $\mathbf{V c}(\mathbf{m} / \mathbf{m i n})$ & $\mathbf{a p}(\mathbf{m m})$ & $\mathbf{f}(\mathbf{m m} / \mathbf{r e v})$ & $\mathbf{R a}(\boldsymbol{\mu} \mathbf{m})$ & $\mathbf{R z}(\boldsymbol{\mu m})$ \\
\hline 1 & 179,79 & 2 & 0,19 & 7,05 & 36,18 \\
\hline 2 & 176,62 & 2 & 0,19 & 7,53 & 40,39 \\
\hline 3 & 173,59 & 2 & 0,19 & 7,53 & 40,30 \\
\hline 4 & 170,41 & 2 & 0,19 & 7,92 & 41,00 \\
\hline 5 & 167,46 & 2 & 0,19 & 8,32 & 43,15 \\
\hline 6 & 164,40 & 2 & 0,19 & 8,15 & 44,59 \\
\hline 7 & 161,32 & 2 & 0,19 & 8,07 & 42,86 \\
\hline 8 & 158,31 & 2 & 0,19 & 9,90 & 48,05 \\
\hline 9 & 155,19 & 2 & 0,19 & 10,16 & 48,99 \\
\hline 10 & 152,12 & 2 & 0,19 & 10,64 & 49,96 \\
\hline 11 & 188,31 & 2 & 0,19 & 9,35 & 49,75 \\
\hline 12 & 184,57 & 2 & 0,19 & 6,59 & 33,90 \\
\hline 13 & 180,82 & 2 & 0,19 & 7,99 & 38,45 \\
\hline 14 & 177,37 & 2 & 0,19 & 6,81 & 32,47 \\
\hline 15 & 173,77 & 2 & 0,19 & 8,53 & 45,95 \\
\hline 16 & 170,50 & 2 & 0,19 & 21,27 & 118,50 \\
\hline
\end{tabular}

Este ensaio também apresentou resultados distintos do esperado para os parâmetros de rugosidade média. No início da operação, observou-se que $\mathrm{Ra}$ e $\mathrm{Rz}$ aumentam aos poucos, chegando depois de algumas passadas ao limite permitido. Logo após, houve a redução dos valores de Ra e Rz até o momento que ultrapassou o limite, na qual ocorreu uma falha na ferramenta. Pode ser explicado esse acontecimento devido ao aumento do raio de ponta da ferramenta.

Em um novo ensaio, decidiu-se verificar como a profundidade de corte pode influenciar nos resultados de rugosidade. Logo, a profundidade de corte utilizada foi ap $=2,5 \mathrm{~mm}$ no diâmetro da peça. Este valor é menor do que o máximo recomendado para a ferramenta e maior que a condição dita como ótima pelo fabricante. $\mathrm{O}$ avanço utilizado foi o considerado ótimo pelo fabricante. E a velocidade de corte utilizada em todo ensaio foi ligeiramente maior que a mínima indicada pelo fabricante, próximo do limite de Vc mínimo, $180 \mathrm{~m} / \mathrm{min}$. Com esta profundidade de corte estabelecida, foram realizados oito passes, até se danificar a ferramenta. Durante o ensaio, a velocidade de corte variou de $\mathrm{Vc}=151,52$ a $\mathrm{Vc}=125,82 \mathrm{~m} / \mathrm{min}$.. Os resultados obtidos para este ensaio estão na Tab. 3. Nota-se que a rugosidade média se manteve em um estado muito menor do que o limite, aproximadamente a metade do valor limite de Ra. Destaca-se que estes resultados são válidos somente para o aço ABNT 1020 e as condições de corte estabelecidas nos ensaios.

Tabela 3: Avaliação da profundidade de corte.

\begin{tabular}{|l|l|l|l|l|l|}
\hline Passe & Vc $(\mathbf{m} / \mathbf{m i n})$ & $\mathbf{a p}(\mathbf{m m})$ & $\mathbf{f}(\mathbf{m m} / \mathbf{r e v})$ & $\mathbf{R a}(\boldsymbol{\mu m})$ & $\mathbf{R z}(\boldsymbol{\mu m})$ \\
\hline 1 & 151,52 & 2,5 & 0,19 & 6,57 & 33,7 \\
\hline
\end{tabular}




\begin{tabular}{|l|l|l|l|l|l|}
\hline 2 & 147,26 & 2,5 & 0,19 & 6,62 & 35,01 \\
\hline 3 & 143,38 & 2,5 & 0,19 & 6,71 & 35,35 \\
\hline 4 & 140,09 & 2,5 & 0,19 & 6,86 & 35,59 \\
\hline 5 & 135,94 & 2,5 & 0,19 & 7,04 & 37,84 \\
\hline 6 & 132,45 & 2,5 & 0,19 & 6,73 & 35,93 \\
\hline 7 & 128,45 & 2,5 & 0,19 & 6,36 & 34,42 \\
\hline 8 & 125,82 & 2,5 & 0,19 & 31,13 & 151,56 \\
\hline
\end{tabular}

\section{CONCLUSÕES}

Com os resultados alcançados nos ensaios, verifica-se que dentre os parâmetros de corte ensaiados, a velocidade de corte e a profundidade de corte exerceram as maiores influencias nos resultados de rugosidade das superfícies usinadas.

Para os avanços utilizados, $0,19 \mathrm{~mm} / \mathrm{rev}$ e o de $0,348 \mathrm{~mm} / \mathrm{rev}$, o de $0,19 \mathrm{~mm} / \mathrm{rev}$ obteve os melhores desempenhos. Para a profundidade de corte utilizada, de $2 \mathrm{~mm}$ e de $2,5 \mathrm{~mm}$, a de $2 \mathrm{~mm}$ obteve o melhor desempenho. As velocidades de corte utilizadas obtiveram ótimos desempenhos.

\section{REFERÊNCIAS}

FREITAS, M. T. T., "Estudo das condições de corte no resultado de trabalho do processo de torneamento do aço abnt 1020" , Ttrabalho de conclusão de curso em Engenharia Mecânica, Universidade federal de Viçosa, 2015.

AMORIM, H. J., "Estudo da relação entre velocidade de corte, desgaste de ferramenta, rugosidade e forças de usinagem em torneamento com ferramenta de metal duro", Dissertação (Mestrado em Engenharia Mecânica) - Universidade Federal do Rio Grande do Sul, Porto Alegre, 2002.

BENINI, L., "Manufatura Assistida por Computador". Departamento de Engenharia Mecânica da UFV. 2015. Notas de Aula. 